\title{
BMJ Open Meanings of 'centredness' in long-term care facilities: a scoping review protocol
}

\author{
Genevieve Thompson, ${ }^{1}$ Chloe Lyn Shindruk, ${ }^{1}$ Adebusola Abiodun Adekoya, ${ }^{1}$ \\ Lisa Demczuk, ${ }^{2}$ Susan McClement ${ }^{1}$
}

To cite: Thompson $\mathrm{G}$, Shindruk CL, Adekoya AA, et al. Meanings of 'centredness' in long-term care facilities: a scoping review protocol. BMJ Open 2018;8:e022498. doi:10.1136/ bmjopen-2018-022498

- Prepublication history and additional material for this paper are available online. To view these files, please visit the journal online (http://dx.doi org/10.1136/bmjopen-2018022498).

Received 22 February 2018 Revised 25 May 2018 Accepted 3 July 2018

Check for updates

(C) Author(s) (or their employer(s)) 2018. Re-use permitted under CC BY-NC. No commercial re-use. See rights and permissions. Published by BMJ.

${ }^{1}$ College of Nursing, Rady Faculty of Health Sciences, University of Manitoba, Winnipeg, Manitoba, Canada ${ }^{2}$ Elizabeth Dafoe Library, University of Manitoba, Winnipeg, Manitoba, Canada

Correspondence to Dr Genevieve Thompson; genevieve.thompson@ umanitoba.ca

\begin{abstract}
Introduction There is a growing demand for long-term care services for older adults that embrace a model of care centred on individual recipients of care. In longterm care, models of person, resident and relationshipcentred care have been developed and implemented to promote independence, decision making and choices of residents. Although the concepts of centredness have been readily adopted in these environments, what constitutes centredness is often vague and lacks conceptual clarity and definition. The research questions guiding this scoping review are: (1) What are the defining attributes, conceptual boundaries and theoretical underpinnings of each centredness term in long-term care? (2) For what purposes have centredness directed models of care been used in this context? (3) What types of study designs have been used to examine centredness in this context? (4) What outcomes related to centredness have been reported or evaluated and how were they measured in long-term care?
\end{abstract}

Methods and analysis This review uses the methodological framework for conducting a scoping review by Arksey and 0'Malley. The search strategy will be applied to nine bibliographic and citation databases, Google Scholar and the grey literature. Study selection will occur in a two-step process. First, the titles and abstracts of all search results will be screened by individual reviewers. Second, a full-text review will be conducted by a pair of reviewers. To be included articles must (1) define centredness in the context of long-term care; (2) describe the defining features of centredness; (3) explore the theoretical underpinnings of centredness; (4) outline outcomes of centredness or (5) use outcome measures related to centredness. Data will be extracted from included studies and analysed using thematic analysis as described by Braun and Clark.

Ethics and dissemination Research ethics approval is not required for this scoping review. Dissemination strategies will follow a targeted and tailored approach based on study findings.

Over the past several decades, there has been a concerted effort in the health sciences to become more 'centred' in the development and delivery of healthcare. ${ }^{1}$ Espoused as a means to increase the quality of healthcare delivery $^{2}$ and to improve the social, psychological and ethical sensitives of healthcare providers in their interactions with patients
Strengths and limitations of this study

Being a scoping rather than a systematic review, the quality of the evidence will not be evaluated.

- The review will follow a validated framework, the search strategy will be developed with librarian expertise, and a systematic approach to data analysis will be used.

- This review offers a targeted view of how centredness terms have been used specifically in long-term care and will provide conceptual clarity useful for decision makers, researchers and clinicians.

- Various centredness outcomes will be included and the variables they purport to change will be reported, providing guidance for future research.

- The review will be limited to English language studies.

and families, ${ }^{3}$ being 'centred' has come to be seen as a core value in healthcare. As a concept, centredness has increasingly been used to guide health and social policies, clinical practice and research. ${ }^{4-6}$ While it seems a reasonable assertion that the concept of 'centredness' guide practice across settings of care, ${ }^{7}$ it is a 'fuzzy concept ${ }^{38}$; increasingly so because of the plethora of concepts that it encompasses that are often used interchangeably without consideration of the theoretical or philosophical foundations in which they are grounded. Achieving a sense of understanding of the defining attributes, characteristics and outcomes of the various 'centredness' terms will address this concept in a way that is 'not simply a matter of semantics but instead it is about establishing greater conceptual and theoretical clarity.' (McCormack et al, p621) ${ }^{7}$ A rigorous examination of centredness will assist to understand its meaning, to gain conceptual clarity of these various terms and to determine whether these terms can be used interchangeably or if they represent distinct concepts; all of which are critical steps in advancing the state of the science forward. 


\section{BACKGROUND}

Centredness is the overarching umbrella term to represent person-centred, resident-centred, patient-centred, family-centred and relationship-centred care concepts. Depending on the setting in which care is provided, the term used may vary. For example, patient-centred care is most frequently invoked in acute care settings, whereas in long-term care settings, the terms person-centred, resident-centred and relationship-centred care are more often used. Family-centred care appears most frequently related to the context of obstetrical and critical care.

The need to promote centredness in healthcare, especially in long-term care settings, has been well established in the literature. ${ }^{9}$ For the purpose of this scoping review, long-term care facilities, often known as nursing or care homes, can be understood as providing living accommodation for people who require on-site delivery of 24 hours, 7 days a week supervised care, including professional health services, personal care and services such as meals, laundry and housekeeping. ${ }^{10}$ While not all residents of long-term care facilities are older adults, only about $6.7 \%$ of Canadian residential long-term care residents are younger than 65 years. ${ }^{11}$ Individuals living in long-term care facilities require complex care as most have considerable needs related to multiple medical diagnoses and over half of residents have a diagnosis of an age-related dementia. ${ }^{12}$

With an increase in the ageing population, there is a growing demand for long-term care services for older adults. However, there is a mounting concern that people living in long-term care facilities are not receiving the quality of care they require and as a result are socially isolated, helpless and bored. ${ }^{13} 14$ People living in longterm care facilities are often reported to have lower quality of life and poorer health outcomes than those living in the community. ${ }^{13}$ Many long-term care facilities operate using the traditional medical model where people are often medicalised, subjected to ageism and have little or no power and control over their lives. ${ }^{15} 16$

Several studies have suggested the need for culture change in long-term care facilities by shifting away from the medical model and de-institutionalising the environment and care practices. ${ }^{131517}$ Person-centred care has been recommended as the foundation for culture change in long-term care facilities and for the promotion of a best practice approach in providing quality care. ${ }^{17}$ Healthcare providers are encouraged to centre on the people they provide care for by promoting their independence and their rights to make decisions and choices related to their day-to-day activities. In particular, person-centred practices that focus on the person rather than the disease are crucial in the care of people living with dementia. ${ }^{131819}$ Although the concepts of centredness have been readily adopted by many long-term care facilities, what constitutes centredness is often vague and lacks conceptual clarity and definition. Previous studies have reported confusion around the implementation of some of these concepts. ${ }^{1320}$ Frequently, the use of person-centred, resident-centred, relationship-centred and family-centred care are used interchangeably or are reported side-by-side. ${ }^{7}$ This mixing of terminology fails to acknowledge theoretical, philosophical or conceptual differences that may exist among the terms or even if they result in the same outcomes. As such, there is a muddling of the concepts that does little to advance knowledge and best practices. Therefore, it is imperative to examine the meaning of centredness in the context of long-term care. The objectives of this scoping review are (1) to map the existing literature examining the use of 'centredness' terms in the context of long-term care; (2) to determine the defining conceptual features and outcomes of each and (3) to identify gaps for future research.

\section{METHODS/DESIGN}

After considering the various systematic approaches to reviewing and assessing published literature, we decided to use the scoping review method in order to most broadly capture the current discourse on this topic. Scoping reviews studies are undertaken to map the range of evidence in a field of study and may help to point out gaps in the existing literature. ${ }^{21}$ However, unlike systematic reviews or meta-analyses, the quality of included studies is not evaluated. ${ }^{22}$

To assist in the development of the protocol for the scoping review, we used Arksey and O'Malley's ${ }^{23}$ framework and have incorporated the improvements put forth by Levac and colleagues ${ }^{22}$ to improve the rigour and consistency of the process. This framework proposes five mandatory stages which are outlined below. An optional sixth stage (consultation with stakeholders) is proposed, but our current study has not examined this due to the nascent stage of the project. Once the review is complete, we fully intend to engage in an active knowledge translation stage which will involve consultation with stakeholders.

\section{PATIENT AND PUBLIC INVOLVEMENT}

In terms of stakeholder involvement and consultation, it may be helpful to note that this research question was born out an agenda of research involving collaboration between groups of clinicians, policy makers and academics, but public and patients were not expressly consulted in the development of this protocol.

\section{STAGE 1: IDENTIFYING THE RESEARCH QUESTIONS}

In order to guide the scoping study, broad research questions were developed, and a clearly articulated scope of inquiry was outlined. ${ }^{22}$ As such, we considered the target population, context and centredness frameworks in defining our questions, and subsequent search strategy (see table 1).

As our intention is to compare and contrast common 'centredness' terms used in long-term care and to provide clarification of the definitions and conceptual boundaries 
Table 1 Concepts in relation to the research question

\begin{tabular}{lll}
\hline Population & Context & 'Centredness' frameworks of: \\
\hline Residents & Long-term care & Resident-centred \\
Family caregivers & Nursing homes/nursing home & Person-centred \\
Staff & $\begin{array}{l}\text { Personal care home/personal care } \\
\text { homes }\end{array}$ & Patient-centred \\
Leadership/Management & Aged care facility/aged care home & Family-centred \\
$\begin{array}{l}\text { Older adult } \\
\text { Elder } \\
\text { Senior }\end{array}$ & Homes for the aged & Relationship-centred \\
\hline
\end{tabular}

between these terms, we chose the following specific questions to guide our review:

1. What are the defining attributes, conceptual boundaries and theoretical underpinnings of each centredness term in long-term care facilities?

2. For what purposes have centredness directed models of care been used in the context of long-term care facilities?

3. What types of study designs have been used to examine centredness in long-term care facilities?

4. What outcomes related to centredness in long-term care facilities have been reported or evaluated and how were they measured?

\section{STAGE 2: IDENTIFYING RELEVANT STUDIES}

As a means to ensure comprehensiveness in identifying primary studies and other relevant literature, ${ }^{23}$ the team iteratively decided on criteria for eligibility, databases to search and key terms to be used in the search strategy. While as previously noted, we recognise not all residents of long-term care facilities are older adults (eg, 65 years and older), in many countries, separate facilities are provided for children and young adults; the idea being that while their healthcare needs may be similar, their social and recreational needs are not. ${ }^{24}$ Such facilities that specialise in the care of children and young adults are not the focus of this review.

\section{Eligibility criteria}

The following inclusion criteria guided the search strategy development and will be used for screening studies for inclusion in the review:

i. English language publications.

ii. Years of publication: 1990-current.

iii. Concepts discussed in context of long-term care facilities.

iv. All types of quantitative and qualitative evidence including but not limited to: randomised or quasi-randomised controlled trials; mixed methods studies; qualitative studies; prospective, longitudinal or retrospective case-control studies; systematic reviews, meta-analyses, meta-syntheses, narrative reviews, scoping reviews and general review articles; clinical practice guidelines, consensus statements and relevant reports or policy documents.

v. Evidence included in the review must: (1) define centredness; (2) describe the attributes/defining features of centredness; (3) explore the theoretical and philosophical underpinnings of centredness; (4) outline outcomes of centredness or (5) use outcome measures related to centredness.

Articles will be excluded if 'centredness' is not a central concept within the document such as when the term simply appears as a statement in the Discussion section. Not being published in the English language or not within the context of long-term care will also be excluded.

\section{Databases}

The following nine bibliographic and citation databases have been identified for searching for published studies: MEDLINE (Ovid), the Cumulative Index to Nursing and Allied Health Literature (CINAHL) (EBSCO), PsycINFO (ProQuest), AgeLine (EBSCO), Embase (Ovid), Sociological Abstracts (ProQuest), Web of Science (Thompson Reuters), Social Services Abstracts (ProQuest) and Cochrane Library (Wiley). A review of existing reviews or protocols was done in Cochrane Library (Wiley), Joanna Briggs Institute Database of Systematic Reviews (Ovid), Health Evidence, Turning Research Into Practice (TRIP) database, National Insititue for Health Care (NICE) Evidence, PROSPERO, Campbell Collaboration and the Evidence for Policy and Practice Information (EPPI) Centre. A limited Google Scholar search will be done to identify studies not retrieved by the databases. Grey literature searches will be conducted for dissertations in Dissertations \& Theses (ProQuest) and for other unpublished studies using Open Grey and Grey Literature Report. In addition, targeted searching of identified websites and a limited internet search using Google will be conducted. Hand searching of reference lists and forward citations from included studies will also be performed.

\section{Search strategy}

The librarian on our team provided her expertise in developing the search strategies. Working with other members of the team, she assisted in determining and 
testing keywords, MeSH terms and filters to maximise sensitivity and specificity within the search. A pilot search was conducted of the MEDLINE and CINAHL databases to identify additional relevant keywords and subject headings. A comprehensive search strategy was then developed for MEDLINE and was tested for known key papers. The search is limited to English language papers and publications dates of 1990 to date of the search. Key terms of the search are described in table 1. Attention to the spelling of 'centered' versus 'centred' was made by running each term (eg, person-centered and person-centred). The proposed final search strategy for MEDLINE is provided in the online supplementary appendix A. The strategy will be translated for each database to be searched. As not all of the nine databases are MeSH heading capable, the translation process will involve using a combination of synonymous keywords based, in part, on the categories of exploded $\mathrm{MeSH}$ terms in those databases that are MeSH capable. In addition, special attention will be paid to capturing concepts when possible through use of keywords and terms in database-specific controlled vocabulary searches when MeSH headings are not available, and to the function of and response to Boolean operators in each database. References from the conducted searches will be exported to a reference management system where duplicates will be removed.

\section{STAGE 3: STUDY SELECTION}

Study selection will occur in a two-step process. First, the titles and abstracts of all papers retrieved by the searches will be screened by individual reviewers on the team, to determine eligibility based on the inclusion and exclusion criteria. In order to ensure the screening criteria were being equally applied, the four reviewers will independently assess the first 50 articles and then meet to discuss their conclusions.
The second stage of the process will involve a full-text review for eligibility by two authors (GT, SM, BA, CS), with disagreements resolved through discussion until a consensus is reached. A flow diagram detailing article selection process will be displayed to enhance the transparency about the search and screening process.

\section{STAGE 4: CHARTING THE DATA}

During this stage, data will be extracted from the included studies, using a data extraction form jointly developed by the research team. ${ }^{22}$ Data to be extracted will differ depending on the type of publication but will include standard bibliographic information such as author, title, year of publication and type of evidence as well as definition of 'centredness' and use of theory. A summary of key theoretical and philosophical foundations will be recorded. For primary studies, data will also include study objectives, sample size, study design, outcomes, outcome measures and key findings. ${ }^{25}$ A preliminary data extraction form is provided in table 2 .

Following the recommendations of Levac and colleagues, ${ }^{22}$ the data extraction form will be trialled with the first 10 studies to ensure data extraction is consistent with the research questions and purpose, and that the researchers are consistent in their approach. Data will be entered into Microsoft Excel.

\section{STAGE 5: COLLATING, SUMMARISING AND REPORTING THE RESULTS}

The strength of the scoping review process is in its ability capture the complexity of data in an area and to present an overview that also considers the implications for practice, policy and research. ${ }^{26}$ Levac and colleagues ${ }^{22}$ advocate for a rigorous approach to analysis that involves both a descriptive numerical summary and a thematic analysis of the data.

Table 2 Data extraction framework

\begin{tabular}{lll}
\hline Bibliometrics & Characteristics of primary studies & $\begin{array}{l}\text { Characteristics of reviews and or theoretical/ } \\
\text { philosophical papers }\end{array}$ \\
\hline $\begin{array}{l}\text { Authors } \\
\text { Year of publication }\end{array}$ & Aims/purpose & Aims/purpose \\
\hline Title & Concept(s) & Methodology \\
Source & Definitions of concept(s) & Concept(s) \\
Country & Attributes/defining features of the concepts & Definitions of concept(s) \\
& $\begin{array}{l}\text { Theoretical, philosophical foundations of centredness } \\
\text { term, including primary source of theory and summary } \\
\text { of theory }\end{array}$ & $\begin{array}{l}\text { Attributes/defining features of the concepts } \\
\text { primary source of theory and summary of theory }\end{array}$ \\
& Methodology & Key findings \\
\hline Intervention type and comparator (if applicable) & \\
& Outcomes & \\
& Outcome measures & \\
\hline
\end{tabular}

Characteristics of reviews and or theoretical/ philosophical papers

Aims/purpose

Concept(s)

Definitions of concept(s)

Attributes/defining features of the concepts

Theoretical, philosophical foundations including

Key findings

Intervention type and comparator (if applicable)

\section{Outcome measures}


This approach has been supported by others as a means of strengthening the scoping review methodology. ${ }^{21}$ While this stage of our project is still a work in progress, we have the qualitative expertise on our team to use the thematic approach described by Braun and Clarke ${ }^{27}$ to capture the broad themes in the data. We anticipate using text and visual approaches to presenting our data. More specifically, findings will be displayed through the development of a conceptual map outlining the meanings of each term, the purpose for which they have been used, the theoretical and philosophical underpinnings and the various outcome measures that have been used for each.

\section{ETHICS AND DISSEMINATION}

All data generated from the review will be stored on password-protected computers. Dissemination plans will follow a targeted and tailored approach, as advocated by the Canadian Health Services Research Foundation Model. ${ }^{28}$ Publication of findings in peer-reviewed journals and at national and international conferences will be pursued. Findings relevant to stakeholders such as long-term care administrators, clinicians and policy planners will be presented through webinars, face-to-face meetings and other social media tools.

\section{CONCLUSION}

As healthcare has moved towards a perspective of inclusivity, there has been a multitude of terms to describe this approach to care. The context of long-term care is no exception, with the use of many different 'centredness' terms applied to care philosophies and care provision. However, there has been no rigorous exploration of the theoretical, conceptual or defining features of these terms and the outcomes they purport to impact. Our protocol for systematically conducting a scoping review in this area is a novel approach to knowledge synthesis and aims to provide unique insight into these terms. Understanding this landscape will foster greater clarity in understanding the practice environment in long-term care and identify gaps in our knowledge; gaps that may give direction to exciting and new research to guide clinical practice.

Contributors GT: conceptualised this study and funded the study through her Canadian Institutes for Health Research (CIHR) award. She led and designed this study. CLS, SMC, AAA, LD: assisted with the study design and developing the search strategy. All authors contributed to the manuscript drafts and reviewed the final manuscript.

Funding This work was supported by the CIHR New Investigator Award (grant number FRN122782).

Competing interests None declared.

Patient consent Not required.

Ethics approval Research ethics approval is not required for this scoping review.

Provenance and peer review Not commissioned; externally peer reviewed.

Open access This is an open access article distributed in accordance with the Creative Commons Attribution Non Commercial (CC BY-NC 4.0) license, which permits others to distribute, remix, adapt, build upon this work non-commercially, and license their derivative works on different terms, provided the original work is properly cited, appropriate credit is given, any changes made indicated, and the use is non-commercial. See: http://creativecommons.org/licenses/by-nc/4.0/.

\section{REFERENCES}

1. Sheikh K, Ranson MK, Gilson L. Explorations on people centredness in health systems. Health Policy Plan 2014;29:ii1-5.

2. Medicine I of. Crossing the quality chasm. Washington, D.C: National Academies Press, 2001.

3. Pluut B. Differences that matter: developing critical insights into discourses of patient-centeredness. Med Health Care Philos 2016;19:501-15.

4. Constand MK, MacDermid JC, Dal Bello-Haas V, et al. Scoping review of patient-centered care approaches in healthcare. BMC Health Serv Res 2014;14:271.

5. Arora NK, Gayer C, DiGioia K, et al. A patient-centered approach to research on palliative care for patients with advanced illnesses and their caregivers. J Pain Symptom Manage 2017;54:e1-8.

6. Maher S. The Francis report --the importance of person-centred health and care. Dent Update 2015;42:210-2.

7. McCormack B, Karlsson B, Dewing J, et al. Exploring personcentredness: a qualitative meta-synthesis of four studies. Scand J Caring Sci 2010;24:620-34.

8. Hughes JC, Bamford C, May C. Types of centredness in health care: themes and concepts. Med Health Care Philos 2008;11:455-63.

9. Ruggiano N, Edvardsson D. Person-centeredness in home- and community-based long-term care: current challenges and new directions. Soc Work Health Care 2013;52:846-61.

10. Government of Canada. Long-term facilities-based care. 2004. https://www.canada.ca/en/health-canada/services/home-continuingcare/long-term-facilities-based-care.html (accessed 22 May 2018).

11. Canadian Institute for Health Information. CCRS Profile of Residents in Continuing Care facilities 2016-2017. Quick Stats 2018 https:// www.cihi.ca/en/quick-stats (accessed 22 May 2018).

12. Estabrooks CA, Poss JW, Squires JE, et al. A profile of residents in prairie nursing homes. Can J Aging 2013;32:223-31.

13. Li J, Porock D, Li J. Resident outcomes of person-centered care in long-term care: a narrative review of interventional research. Int J Nurs Stud 2014;51:1395-415.

14. Nolan MR, Davies S, Brown J, et al. Beyond person-centred care: a new vision for gerontological nursing. J Clin Nurs 2004;13:45-53.

15. Harrison J, Frampton S. Resident-Centered Care in 10 U.S. Nursing Homes: Residents' Perspectives. J Nurs Scholarsh 2017;49:6-14.

16. Scales K, Bailey S, Middleton J, et al. Power, empowerment, and person-centred care: using ethnography to examine the everyday practice of unregistered dementia care staff. Sociol Health IIIn 2017;39:227-43.

17. Zimmerman S, Shier V, Saliba D. Transforming nursing home culture: evidence for practice and policy. Gerontologist 2014;54:S1-5.

18. Edvardsson D, Innes A. Measuring person-centered care: a critical comparative review of published tools. Gerontologist 2010;50:834-46.

19. Edvardsson D, Watt E, Pearce F. Patient experiences of caring and person-centredness are associated with perceived nursing care quality. J Adv Nurs 2017;73:217-27.

20. Morgan S, Yoder LH. A concept analysis of person-centered care literature review. J Holist Nurs 2012;30:6-15.

21. Daudt HM, van Mossel C, Scott SJ. Enhancing the scoping study methodology: a large, inter-professional team's experience with Arksey and O'Malley's framework. BMC Med Res Methodol 2013;13:48.

22. Levac D, Colquhoun $\mathrm{H}$, O'Brien KK. Scoping studies: advancing the methodology. Implement Sci 2010;5:69.

23. Arksey H, O'Malley L. Scoping studies: towards a methodological framework. Int J Soc Res Methodol 2005;8:19-32.

24. Banerjee A. An overview of long-term care in Canada and selected provinces and territories. Toronto, 2007. (accessed 22 May 2018).

25. Peters M, Godfrey C, Mclnerney P, et al. Methodology for JBI scoping reviews. The Joanna Briggs Institute reviewers' manual. Adelaide, South Australia, 2015.

26. Colquhoun HL, Levac D, O'Brien KK, et al. Scoping reviews: time for clarity in definition, methods, and reporting. J Clin Epidemiol 2014;67:1291-4.

27. Braun V, Clarke V. Qualitative research in psychology using thematic analysis in psychology using thematic analysis in psychology. Qual Res Psychol 2006;3:77-101.

28. Canadian Health Services Research Foundation. Developing a dissemination plan. http://www.cfhi-fcass.ca/Migrated/PDF/ CommunicationNotes/dissemination_plan_e.pdf (accessed 15 Feb 2018). 